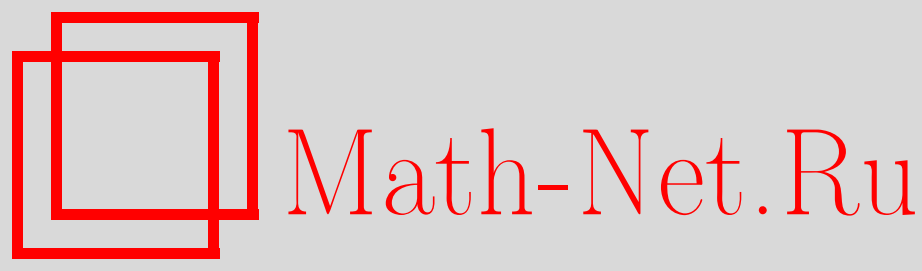

А. М. Магомедов, Т. А. Магомедов, Последовательное разбиение ребер двудольного графа на паросочетания, Дискрет. матем., 2016, том 28, выпуск 1, 78-86

DOI: https://doi.org/10.4213/dm1358

Использование Общероссийского математического портала Math-Net.Ru подразумевает, что вы прочитали и согласны с пользовательским соглашением http://www . mathnet.ru/rus/agreement

Параметры загрузки:

IP : 34.227 .88 .159

26 апреля 2023 г., $12: 30: 13$

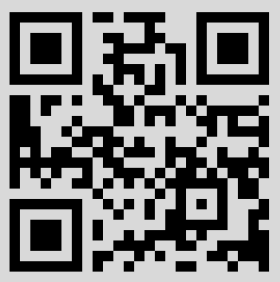




\title{
Последовательное разбиение ребер двудольного графа на паросочетания
}

\author{
() 2016 г. А. М. Магомедов*, Т. А. Магомедов**
}

Пусть исходные данные к расписанию обслуживания множества требований в некоторой системе приборов заданы двудольным графом. Рассматривается задача составления расписания минимальной длины, удовлетворяющего условиям беспростойной работы каждого прибора и их одновременного ввода в работу. Получены условия разбиения множества ребер графа на паросочетания, образующие расписание требуемого вида.

Работа выполнена при финансовой поддержке 1) проекта № 2014/33 в рамках базовой части государственного задания Минобрнауки России, 2) проекта 3c Даггосуниверситета, 3) Отдела математики и информатики ДНЦ РАН.

Ключевые слова: граф, расписание, двудольный, паросочетание, обслуживание

\section{1. Введение}

В статье использованы обозначения и определения книги [1]. Исходные данные к расписанию обслуживания в заданной системе «приборов» $X$ множества «требований» $Y$ представлены двудольным графом $G=(X, Y, E)$. Прибору $x_{i}$ планируется выполнение операции над требованием $y_{j}$ тогда и только тогда, когда множество ребер $E$ содержит ребро $\left(x_{i}, y_{j}\right)$. Одновременное участие объекта (прибора/требования) в двух и более операциях запрещено, длительность каждой операции равна 1, порядок выполнения операций безразличен, отношение частичного предшествования отсутствует. Построить расписание означает тем или иным способом указать для каждого ребра $\left(x_{i}, y_{j}\right) \in E$ промежуток времени единичной длины, когда прибор $x_{i}$ обслуживает требование $y_{j}$.

Биекция множества пронумерованных $(0,1, \ldots)$ дискретных промежутков времени

$$
(0,1],(1,2], \ldots
$$

в множество цветов с аналогичными номерами сводит задачу построения расписания к задаче о правильной реберной раскраске графа. Задача о расписании минимальной длительности эквивалентна задаче о правильной реберной раскраске графа

* Место работы: Дагестанский государственный университет, e-mail: magomedtagir1@yandex.ru

** Место работы: Twitter Inc., e-mail: tagir.magomedov@gmail.com 
$G$ в цвета $0,1, \ldots, \chi^{\prime}(G)-1$, где $\chi^{\prime}(G)$ - реберное хроматическое число графа $G$. Известный результат Г. Визинга [2] гласит, что в общем случае $\chi^{\prime}(G)$ равно $\Delta(G)$ или $\Delta(G)+1$, где через $\Delta(G)$ обозначена наибольшая степень вершины графа $G$. I. Holyer доказал [3], что задача проверки равенства $\chi^{\prime}(G)=\Delta(G)$ NP-полна даже для случая кубических графов. Для случая двудольного графа, согласно теореме Д. Кенига о реберной раскраске [4, с. 80], справедливо равенство $\chi^{\prime}(G)=\Delta(G)$. Поэтому всюду в дальнейшем под расписанием, соответствующим графу $G$, будем подразумевать расписание длительности $\Delta(G)$. Расписание можно определить как разбиение множества $E$ на $\Delta(G)$ паросочетаний множества $X$ с множеством $Y$ :

$$
E_{0}, E_{1}, \ldots, E_{\Delta(G)-1}
$$

где паросочетание $E_{i}$ задает информацию о взаимодействии объектов в момент времени $i ; 0 \leqslant i \leqslant \Delta(G)-1$.

Теорема Д. Кенига о реберной раскраске доказывает существование расписания, но не предлагает способ построения расписания с теми или иными свойствами, полезными для приложений. Особый интерес для приложений представляют такие расписания, где все приборы работают непрерывно с момента одновременного включения, причем прибор $i$ выключается точно по истечении $d_{G} x_{i}$ единиц времени. Другими словами, такие разбиения (1) множества ребер графа $G=(X, Y, E)$, где для каждой вершины $x_{i} \in X$ степени $k$ каждое из паросочетаний $E_{0}, E_{1}, \ldots, E_{k-1}$ содержит ребро, инцидентное вершине $x_{i}$. Разбиение $(1)$, обладающее таким свойством, будем называть последовательным.

На рис. 1 исходные данные к расписанию представлены в виде двудольного графа $G=(X, Y, E)$ с $\Delta(G)=4$. Справа приведено табличное представление соответствующего расписания $T$ длительности 4: если $T_{i k}=j$, то в момент времени $k$ прибор $i$ обслуживает требование $j$. Все приборы включаются одновременно и работают без простоев.

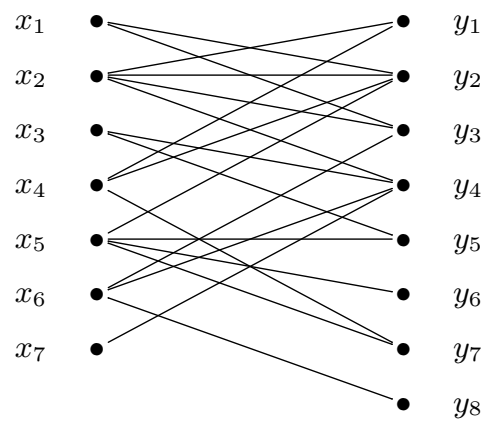

\begin{tabular}{|l|l|l|l|l|}
\hline & $\mathbf{0}$ & $\mathbf{1}$ & $\mathbf{2}$ & $\mathbf{3}$ \\
\hline $\mathbf{1}$ & 3 & 2 & & \\
\hline $\mathbf{2}$ & 2 & 1 & 3 & 4 \\
\hline $\mathbf{3}$ & 5 & 4 & & \\
\hline $\mathbf{4}$ & 1 & 7 & 2 & \\
\hline $\mathbf{5}$ & 6 & 5 & 7 & 2 \\
\hline $\mathbf{6}$ & 8 & 3 & 4 & \\
\hline $\mathbf{7}$ & 4 & & & \\
\hline
\end{tabular}

Рис.1. Слева - граф исходных данных $G=(X, Y, E)$, справа - расписание с одновременным включением и беспростойной работой всех приборов

В статье получены условия существования последовательного разбиения. Последовательные разбиения востребованы в задачах составления расписаний минимальной длительности с беспростойной работой каждого прибора с момента включения вплоть до его выключения (все приборы включаются одновременно). Например, 
лекторы основного вуза могут совмещать основную работу с дополнительной работой в филиале, и от диспетчера расписания основного вуза требуется составить расписание таким образом, чтобы лекции каждого «совместителя» располагались в начале расписания и проводились без «окон» для лектора.

\section{2. Условия существования полного паросочетания в двудольном графе}

Паросочетание в двудольном графе $G=(X, Y, E)$, насыщающее все вершины $X$, называется полным паросочетанием $X$ с $Y$. Необходимые и достаточные условия существования в двудольном графе $G=(X, Y, E)$ полного паросочетания $X$ с $Y$ формулируются в известной теореме Холла [1, с. 164]:

$$
|A| \leqslant|\Gamma(A)| \quad \forall A \subseteq X
$$

где $\Gamma(A)$ - множество вершин, смежных вершинам $A$.

Проверка условий затрудняется требованием выполнения неравенства (2) для каждого подмножества из $X$. Поэтому имеет смысл поиск условий, допускающих простую проверку. Известно, например [1, с. 165], что в двудольном графе $G=(X, Y, E)$ существует полное паросочетание $X$ с $Y$, если

$$
\min _{x \in X} d_{G} x \geqslant \max _{y \in Y} d_{G} y
$$

Последнее равносильно выполнению для всех вершин $x \in X, y \in Y$ неравенства:

$$
d_{G} x \geqslant d_{G} y
$$

Избыточность условия особенно заметна применительно к двудольному графу из нескольких компонент: соотношения между степенями вершин, принадлежащих разным компонентам графа, очевидно, не должны влиять на существование полного паросочетания.

Лемма 1. Пусть задан двудольный граф $D=(A, B, C)$, где $A$ u $B$ - множества вершин, $C$ - множество ребер. Если для каждого ребра $\left(a_{i}, b_{j}\right) \in C$ справедливо неравенство

$$
d_{D} a_{i} \geqslant d_{D} b_{j}
$$

$m o|A| \leqslant|B|$.

Доказательство. Пронумеруем ребра множества $C: e_{1}, e_{2}, \ldots$ Т. к. ввиду (3) для каждого ребра $e_{k}=\left(a_{i_{k}}, b_{j_{k}}\right)$ выполняется неравенство

$$
d_{D} a_{i_{k}} \geqslant d_{D} b_{j_{k}}
$$

Tо

$$
\frac{1}{d_{D} a_{i_{k}}} \leqslant \frac{1}{d_{D} b_{j_{k}}}
$$


Просуммируем почленно неравенства (4) по всем $k$ и заметим, что в левой части полученного неравенства каждое слагаемое вида

$$
\frac{1}{d_{D} a_{q}}, \quad a_{q} \in A
$$

встречается точно $d_{D} a_{q}$ раз; $q=1,2, \ldots,|A|$. Следовательно, сумма в левой части неравенства равна $|A|$. Соответственно сумма в правой части равна $|B|$.

Теорема 1. В двудолъном графе $G=(X, Y, E)$ существует полное паросочетание $X$ с $Y$, если для каждого ребра $(x, y) \in E(x \in X, y \in Y)$ выполняется неравенство $d_{G} x \geqslant d_{G} y$.

Доказательство. Пусть $A$ - произвольное подмножество множества $X, B$ - множество вершин, смежных вершинам $A, C$ - множество ребер, инцидентных вершинам $A$. Для произвольного ребра $(x, y)$ графа $D=(A, B, C)$ из соотношений

$$
d_{D} x=d_{G} x, \quad d_{G} x \geqslant d_{G} y, \quad d_{G} y \geqslant d_{D} y
$$

получим

$$
d_{D} x \geqslant d_{D} y
$$

Другими словами, выполнены условия леммы 1, следовательно,

$$
|A| \leqslant|B|
$$

Таким образом, для графа $G$ выполнены условия теоремы Холла, следовательно, существует полное паросочетание $X$ с $Y$.

\section{3. Наследуемые свойства и последовательные разби- ения}

Паросочетание в двудольном графе $G=(X, Y, E)$, насыщающее все вершины степени $\Delta(G)$, при этом в множестве $X$ - только вершины степени $\Delta(G)$, будем называть минимаксом графа $G$. Будем говорить, что двудольный граф $G=(X, Y, E)$ обладает свойством жесткой асимметрии, если

$$
d_{G} x \geqslant d_{G} y \text { для каждого ребра }(x, y) \in E, \quad x \in X, y \in Y \text {. }
$$

Лемма 2. Двудольный граф $G=(X, Y, E)$ со свойством жесткой асимметрии обладает минимаксом.

Доказательство. Рассмотрим подграф $G^{\prime}=\left(X^{\prime}, Y^{\prime}, E^{\prime}\right)$ графа $G$, порожденный множеством всех ребер, инцидентных хотя бы одной вершине степени $\Delta(G)$. Из свойства жесткой асимметрии графа $G$ следует, что степени всех вершин множества $X^{\prime}$ в графе $G^{\prime}$ равны $\Delta(G)$. В двудольном графе $G^{\prime}$ имеется паросочетание $M^{\prime}$, насыщающее все вершины наибольшей степени [1, с. 169]. Паросочетание $M^{\prime}$ является минимаксом графа $G$. 
Следствие 1. Пусть двудольный граф $G=(X, Y, E)$ обладает свойством жесткой асимметрии, $x \in X-$ вершина степени $\Delta(G),(x, y)$ - произвольно выбранное ребро, инцидентное вершине $x$. Граф $G$ обладает минимаксом, содержащим ребро $(x, y)$.

Доказательство. Множество ребер $E^{\prime}$ подграфа $G^{\prime}=\left(X^{\prime}, Y^{\prime}, E^{\prime}\right)$ в доказательстве леммы 2 допускает разбиение на $\Delta\left(G^{\prime}\right)$ полных паросочетаний множества $X^{\prime}$ с множеством $Y^{\prime}[1$, с. 169]. В качестве искомого минимакса выберем то из этих полных паросочетаний, которое содержит ребро $(x, y)$.

Для графа со свойством жесткой асимметрии минимакс является, очевидно, минимальным по мощности паросочетанием, насыщающим все вершины максимальной степени. Граф, полученный удалением минимакса из графа $G$ (здесь и далее - «в случае существования»), будем называть потомком 1-го уровня и обозначать через $G_{1}$ (для исходного графа $G$ удобно иногда применять и обозначение $G_{0}$ ); граф, полученный из $G_{1}$ удалением его минимакса, будем называть потомком 2-го уровня и обозначать $G_{2}$, и так далее. Понятно, что $\Delta\left(G_{k}\right)=\Delta(G)-k, k=0,1, \ldots, \Delta(G)-1$. Так как степени всех вершин графа $G_{\Delta}(G)-1$ равны единице, то множество его ребер является минимаксом, который служит для исходного графа $G=(X, Y, E)$ полным паросочетанием множества $X$ с множеством $Y$.

С учетом способа индексации, принятого в определении последовательного разбиения, минимакс потомка $G_{k}$ обозначим через $E_{\Delta(G)-k-1} ;$ при $k=0,1, \ldots, \Delta(G)-1$ получим последовательно $E_{\Delta(G)-1}, \ldots, E_{1}, E_{0}$.

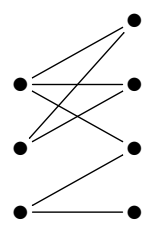

$\Gamma \operatorname{paф} G$ 1

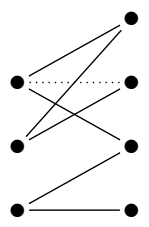

2

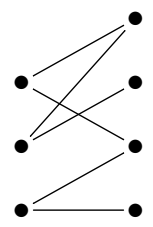

Потомок $G_{1}$ 3

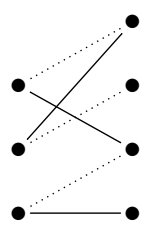

Потомок $G_{2}$

4

5

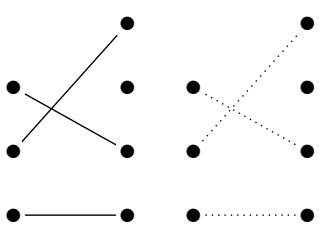

6

Рис. 2. Построение потомков и минимаксов

На рис. 2 приведен пример построения потомков и минимаксов. Граф $G$ на рис. 2.1 обладает свойством жесткой асимметриии, $\Delta(G)=3$; ребро, выделенное на рис. 2.2 , составляет минимакс $E_{2}$, удаление которого приводит к потомку $G_{1}$ (рис. 2.3 ); удаление выделенного на рис. 2.4 минимакса $E_{1}$ графа $G_{1}$ приводит к потомку $G_{2}$ на рис. 2.5 ; ребра $G_{2}$ образуют минимакс $E_{0}$, повторно воспроизведенный на рис. 2.6 из соображений единообразного представления минимаксов.

Пусть $P$ - некоторое свойство двудольного графа $G=(X, Y, E)$, достаточное для существования минимакса у графа $G$. Такое свойство $P$ будем называть наследуемъљм, если выполнение свойства $P$ для графа $G$ влечет выполнение свойства $P$ для каждого потомка 1-го уровня. 
Замечание 1. Если граф $G$ обладает наследуемым свойством, то каждый потомок графа $G$ обладает минимаксом.

Теорема 2. Свойство жесткой асимметрии является наследуемым свойством.

Доказательство. Докажем, что если граф $G=(X, Y, E)$ обладает свойством (5), то и любой его потомок 1-го уровня $G_{1}$ обладает этим свойством. Для любого ребра $(x, y)$ графа $G_{1}$ возможны следующие три случая.

(1) $\mathbf{d}_{G} y=\Delta(G)$. Тогда, в соответствии с $(5), d_{G} x=\Delta(G)$, поэтому $d_{G_{1}} x=d_{G_{1}} y=$ $\Delta(G)-1$.

(2) $\mathbf{d}_{G} y<\Delta(G), d_{G} x=\Delta(G)$. Тогда $d_{G_{1}} x=\Delta(G)-1 \geqslant d_{G} y \geqslant d_{G_{1}} y$.

(3) $\mathbf{d}_{G} y<\Delta(G), d_{G} x<\Delta(G)$. Тогда $d_{G_{1}} x=d_{G} x \geqslant d_{G} y \geqslant d_{G_{1}} y$.

Таким образом, во всех случаях $d_{G_{1}} x \geqslant d_{G_{1}} y$.

Теорема 3. Если двудольный граф $G=(X, Y, E)$ обладает наследуемым свойством, то последовательное разбиение существует.

Доказательство. Пусть граф $G$ обладает наследуемым свойством. В соответствии с замечанием 1 каждый потомок $G_{k}$ обладает минимаксом $E_{\Delta(G)-k-1}, k=$ $0,1, \ldots, \Delta(G)-1$.

Поскольку при каждом $k=0,1, \ldots, \Delta(G)-1$ в $E_{\Delta(G)-k-1}$ насыщены все вершины, имеющие в $G_{k}$ максимальные степени (т. е. $\Delta(G)-k$ ), то в $E_{0}$ насыщены все вершины $X$, в $E_{1}$ - все вершины $X$, степени которых в графе $G$ не меньше $2, \ldots$, в $E_{\Delta(G)-1}$ - все вершины $X$, степени которых в $G$ не меньше $\Delta(G)$ (следовательно, равны $\Delta(G))$. Таким образом, получено последовательное разбиение.

Замечание 2. Из теорем 2 и 3 видно, что для двудольного графа $G=(X, Y, E)$ со свойством жесткой асимметрии существует последовательное разбиение. Данный результат был получен ранее в работе [5] с использованием принципиально другого подхода.

Лемма 3. Пусть для двудольного графа $G=(X, Y, E)$ выполнено свойство жесткой асимметрии, $\lambda u k$ - целые положительные числа, $k>1$, степень некоторой вершины $x_{0}$ множества $X$ равна $\lambda u\left(x_{0}, y^{(0)}\right), \ldots,\left(x_{0}, y^{(k-1)}\right)$ - висячие ребра. Тогда существует такое последовательное разбиение множества $E$ :

$$
E_{0}, E_{1}, \ldots, E_{\Delta(G)-1}
$$

что ребро $\left(x_{0}, y^{(i)}\right)$ участвует в паросочетании $E_{\lambda-i-1}, 0 \leqslant i \leqslant k-1$.

Доказательство. Пусть минимаксы $E_{\Delta(G)-1}, E_{\Delta(G)-2}, \ldots, E_{\lambda}$ вычислены (и удалены). При вычислении каждого минимакса $E_{\lambda-i-1}(0 \leqslant i \leqslant k-1)$ воспользуемся следствием леммы 2 , выбирая каждый раз вместо ребра $(x, y)$ ребро $\left(x_{0}, y^{(i)}\right)$. Затем продолжим вычисление минимаксов $E_{\lambda-k-1}, \ldots, E_{1}, E_{0}$.

Если в графе $G=(X, Y, E)$ имеется такое ребро $\left(x_{0}, y_{0}\right)$, что $d_{G} x_{0}<d_{G} y_{0}$, то ребро $\left(x_{0}, y_{0}\right)$ будем называть дефектом графа $G$.

Лемма 4. Если двудольный граф $G=(X, Y, E)$ имеет единственный дефект, то последовательное разбиение существует. 
Доказательство. Пусть $\left(x_{0}, y_{0}\right)$ - единственный дефект графа $G$,

$$
k=d_{G} y_{0}-d_{G} x_{0}, \quad \lambda=d_{G} y_{0} .
$$

Добавим $k$ новых вершин $y^{(0)}, \ldots, y^{(k-1)}$ к множеству $Y$ и проведем новые ребра $\left(x_{0}, y^{(0)}\right), \ldots,\left(x_{0}, y^{(k-1)}\right)$. Полученный граф $G^{\prime}=\left(X, Y^{\prime}, E^{\prime}\right)$ обладает, очевидно, свойством жесткой асимметрии. По лемме 3 для $E^{\prime}$ существует последовательное разбиение

$$
E_{0}^{\prime}, E_{1}^{\prime}, \ldots, E_{\Delta\left(G^{\prime}\right)-1}^{\prime},
$$

где ребра $\left(x_{0}, y^{(0)}\right), \ldots,\left(x_{0}, y^{(k-1)}\right)$ участвуют в паросочетаниях $E_{\lambda-1}^{\prime}, \ldots, E_{\lambda-k}^{\prime}$ соответственно. Набор паросочетаний:

$$
E_{0}^{\prime}, \ldots, E_{\lambda-k-1}^{\prime}, E_{\lambda-k}^{\prime} \backslash\left\{\left(x_{0}, y^{(k-1)}\right)\right\}, \ldots, E_{\lambda-1}^{\prime} \backslash\left\{\left(x_{0}, y^{(0)}\right)\right\}, E_{\lambda}^{\prime}, \ldots, E_{\Delta\left(G^{\prime}\right)-1}^{\prime}
$$

является последовательным разбиением для множества $E$.

\section{4. Свойство мягкой асимметрии и последовательные разбиения}

Рассмотрим для двудольного графа $G=(X, Y, E)$ следующее свойство:

$$
d_{G} x_{1}+d_{G} x_{2} \geqslant d_{G} y_{1}+d_{G} y_{2} \text { для каждой пары ребер }\left(x_{1}, y_{1}\right) \text { и }\left(x_{2}, y_{2}\right) \text {. }
$$

Свойство (6) будем называть мягкой асимметрией. Очевидно, что (6) следует из (5), но свойство (6) может выполняться и в ситуации, когда (5) нарушено (рис. 3). Граф $G$, изображенный на рис. 3.а, обладает свойством мягкой асимметрии, но не обладает свойством жесткой асимметрии, поскольку $d_{G} x_{1}<d_{G} y_{1}$. Заметим, что при этом минимаксы $E_{0}, E_{1}$ и $E_{2}$ существуют, они приведены на рис. 3.d, 3.c и 3.b соответственно.

$$
\text { Граф G }
$$

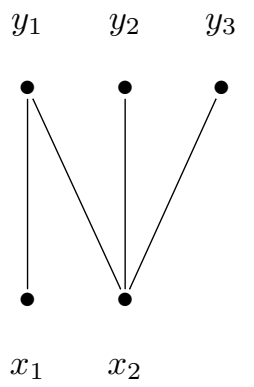

a)

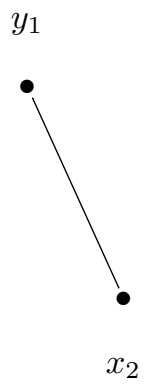

b)

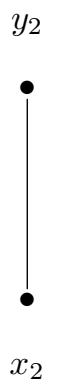

c)

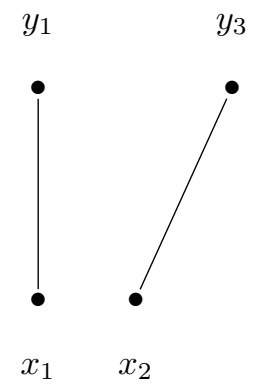

d)

Рис. 3. Для графа $G$ выполнено свойство мягкой асимметрии, но свойство жесткой асимметрии нарушено

Лемма 5. Если двудольный граф $G=(X, Y, E)$ обладает свойством мягкой асимметрии, $y \in Y$ и $d_{G} y=\Delta(G)$, то степень любой вершины $x \in X$, смежной вериине $y$, равна $\Delta(G)$. 
Доказательство. Пусть $x$ - произвольная вершина из $X$, смежная вершине $y$. Если $\Delta(G)=1$, то равенство $d_{G} x=\Delta(G)$ очевидно. Пусть $\Delta(G) \geqslant 2$. Выбрав вершину $x_{1}$, смежную вершине $y$ и отличную от $x$, в соответствии со свойством мягкой асимметрии имеем для ребер $(x, y)$ и $\left(x_{1}, y\right)$ :

$$
d_{G} x+d_{G} x_{1} \geqslant d_{G} y+d_{G} y=2 \Delta(G) .
$$

Отсюда $d_{G} x=d_{G} x_{1}=\Delta(G)$.

Следующая лемма является аналогом леммы 2.

Лемма 6. Двудольньй граф $G=(X, Y, E)$ со свойством мягкой асимметрии обладает минимаксом.

Доказательство. Достаточно повторить доказательство леммы 2, заменив в тексте доказательства предложение «Из свойства жесткой асимметрии графа $G$ следует, что степени всех вершин множества $X^{\prime}$ равны $\Delta(G)$ » на предложение «Из леммы 5 следует, что степени всех вершин множества $X^{\prime}$ равны $\Delta(G) »$.

Граф, изображенный на рис. 4 , обладает свойством мягкой асимметрии, но потомок $G_{1}$, полученный удалением выделенного минимакса $\left\{\left(x_{1}, y_{2}\right),\left(x_{2}, y_{4}\right)\right\}$, не обладает этим свойством, так как для ребер $\left(x_{2}, y_{5}\right)$ и $\left(x_{3}, y_{5}\right)$ соответствующее неравенство нарушено:

$$
d_{G_{1}} x_{2}+d_{G_{1}} x_{3}=5, d_{G_{1}} y_{5}+d_{G_{1}} y_{5}=6 \Rightarrow d_{G_{1}} x_{2}+d_{G_{1}} x_{3}<d_{G_{1}} y_{5}+d_{G_{1}} y_{5} .
$$

Таким образом, свойство мягкой асимметрии не относится к наследуемым свойствам.

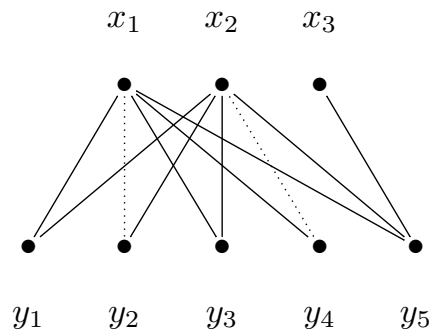

Рис. 4. Граф $G$ обладает свойством мягкой асимметрии, но потомок $G_{1}$ не обладает данным свойством

Лемма 7. Двудольньй граф $G=(X, Y, E)$ со свойством мягкой асимметрии может иметь лишь один дефект.

Доказательство. В самом деле, пусть у графа $G$ имеются два дефекта $\left(x_{1}, y_{1}\right)$ и $\left(x_{2}, y_{2}\right)$ :

$$
d_{G} x_{1}<d_{G} y_{1}, \quad d_{G} x_{2}<d_{G} y_{2} .
$$

Но результат почленного сложения этих двух неравенств противоречит свойству мягкой асимметрии.

Теорема 4. Если двудольный граф $G=(X, Y, E)$ обладает свойством мягкой асимметрии, то последовательное разбиение существует. 
Доказательство. В случае отсутствия дефектов сошлемся на замечание 2. В противном случае имеется в точности один дефект (лемма 7), и согласно лемме 4 последовательное разбиение существует.

\section{5. Заключение}

Перспективным представляется поиск условий существования расписаний рассмотренного выше типа, но без требования одновременного включения приборов. В общем случае задача о существовании таких расписаний является NP-полной [6]. Для одного частного случая условия существования расписания длительности 5 получены в [7], алгоритм их проверки изложен в [8]. Для случая, когда степень каждой вершины множества $X$ равна $\Delta(G), \Delta(G)-2$ или 2, задача рассмотрена в [9].

\section{Список литературы}

1. Свами М., Тхуласираман К., Графы, сети и алгоритмы, Мир, М., 1982; пер. с англ.: Swamy M. N. S., Thulasiraman K., Graphs, Networks, and Algorithms, Wiley, 1981, 592 pp.

2. Визинг В.Г., "Об оценке хроматического класса $p$-графа", Дискретный анализ. Сб. науч. тр., 3, Ин-т математики СО АН СССР, Новосибирск, 1964, 25-30.

3. Holyer I., "The NP-completeness of edge-coloring", SIAM J. Comput., 10:4 (1981), 718-720.

4. Ловас Л., Пламмер М., Прикладные задачи теории графов. Теория паросочетаний в математике, физике, химии, Мир, М., 1998; пер. с англ.: Lovász L., Plummer M.D., Matching theory, North-Holland, 1986, 544 pp.

5. Асратян А. С., Камалян Р. Р., "Интервальные раскраски ребер мультиграфа", Прикладная математика, 5, Изд-во Ереванского ун-та, Ереван, 1987, 25-34.

6. Магомедов А. М., "Непрерывное расписание для специализированных процессоров без отношения предшествования", Вестник МЭИ, сер. Автоматика, вычисл. техника, информатика, 5 (2009), 14-17.

7. Магомедов А. М., Сапоженко А. А., "Условия существования непрерывных расписаний длительности пять", Вестник МГУ, сер. Вычисл. матем. и кибернетика, 34:1 (2010), 39-44; англ. пер.: Magomedov A.M., Sapozhenko A.A., "Conditions for the existence of continuous schedules of duration five", Moscow Univ. Comp. Math. and Cybernetics, 34:1 (2010), 37-43.

8. Магомедов А. М., Магомедов Т.А., "О приложении алгоритма вычисления подграфа максимальной плотности к задаче оптимизации расписания”, Матем.заметки, 93:2 (2013), 313-315; англ. пер.: Magomedov A.M., Magomedov T.A., "Application of an algorithm for calculating the maximum density subgraph to the schedule optimization problem", Math. Notes, 93:2 (2013), 340-342.

9. Магомедов А. М., "Непрерывное учебное расписание с $m, m-2$ или 2 уроками у преподавателей", Дискретная математика, 24:2 (2012), 37-45; англ. пер.: Magomedov A.M., "A continuous timetable with $m, m-2$, or 2 instructor's class hours", Discrete Math. Appl., 22:3 (2012), 261-271. 